\author{
Classification \\ Physics Abstracts \\ 42.30. $\mathrm{Va}-07.07 . \mathrm{Hj}-42.79 . \mathrm{Ci}-85.60 . \mathrm{Gz}-07.60 . \mathrm{Pb}$
}

\title{
Numerical Acquisition of Multiple Immunofluorescence Labelling
}

\author{
Catherine Souchier $\left({ }^{1,2}\right)$, Mehdi Benchaib $\left({ }^{1}\right)$, Richard Delorme $\left({ }^{1}\right)$, \\ Paul André Bryon $\left({ }^{1}\right)$ and Alain Niveleau $\left({ }^{2}\right)$ \\ $\left({ }^{1}\right)$ Cytologie Analytique, 8 av. Rockefeller, 69373 Lyon Cedex 08, France \\ $\left({ }^{2}\right)$ Centre Commun de Quantimétrie, 8 av. Rockefeller, 69373 Lyon Cedex 08, France
}

\begin{abstract}
Résumé. - Cet article présente une évaluation d'outils développés pour l'acquisition numérique d'images de fluorescence. Les filtres double ou triple bande réduisent l'intensité des fluorochromes dont l'émission se situe dans le rouge et le bleu. Limage couleur d'un marquage multiple peut s'obtenir par combinaison d'images monochromes, à condition de vérifier et de corriger si nécessaire l'alignement en $X, Y$ et $Z$. La microscopie confocale présente un intérêt essentiellement pour l'étude d'échantillons épais et l'analyse 3D à fort grossissement. Indépendamment des caractéristiques des caméras, l'interface utilisateur apparaît comme un point clé dans l'acquisition des images.
\end{abstract}

\begin{abstract}
Tools used in numerical acquisition of epi-fluorescence images are evaluated. Using multiple-band filter sets, the intensities of blue and red emitting fluorochromes are reduced. Color images may be reconstructed from monochrome images. Nevertheless, they may have $X / Y$ and $Z$ shifted and need to be realigned before being superimposed. Confocal laser scanning microscope (CLSM) presents an advantage mainly for 3D microscopy of thick specimens. Apart from camera performances, user interface is a key point in image acquisition.
\end{abstract}

\section{Introduction}

Numerical acquisition of multiple immunofluorescence labellings may be performed using an epi-fluorescence microscope fitted either to a confocal laser scanning device (CLSM) or to a monochrome or colour low-light camera (silicon intensified target (SIT), cooled charge coupled device (CCD), intensified CCD) monitored by an image analysis system. All these equipments are currently available. This study reports observations made using the above mentioned techniques.

\section{Material and methods}

Most specimens used during this work were lymphoid cells and metaphases from patients with hematological diseases. They were observed under an epifluorescence microscope (DMRBE, Leica, Germany) using $\times 40 / 1.0$ and $\times 100 / 1.3$ oil PL-Fluotar objectives. Narrow mono-band, dual-band and triple-band fluorescence filter sets (Leica, Germany) were used (Tab. I). Images 
were acquired using a 3CCD colour video camera (DXC-930P, Sony, Japan), fitted to an image analysis system (Quantimet 600, Leica, UK). Images may be time integrated. For colour spreading evaluation, fluorescence intensities were measured on about 40 images per slide. Furthermore, a few acquisitions were performed using monochrome and colour, cooled and intensified, CCD cameras from Lhesa Electronique (France), Hamamatsu (Japan) and Kodak (USA) companies. Two images (Figs. 1B, D) were acquired under another microscope (axioplan, Zeiss, Germany) with similar filter sets except for the blue emission one: band pass (BP) 470/20. Confocal images were acquired under a CLSM (LSM 10, Zeiss, Germany). Fluorochrome excitations were obtained using $\mathrm{HeNe}(\lambda=543 \mathrm{~nm})$, $\operatorname{argon}(\lambda=488 \mathrm{~nm})$, UV argon $(\lambda=364 \mathrm{~nm})$ laser bands. Fluorescence emissions were respectively recorded through a $590 \mathrm{~nm}$ long pass (LP) filter, a $530 / 15 \mathrm{~nm}$ BP filter and a $397 \mathrm{~nm}$ LP filter.

\section{Results and Discussion}

3.1 Colour Acquisition Using Mono or Multiple Band Filter Sets. - In conventional epifluorescence microscopy, colour images of specimens labelled with two or three fluorescent dyes may be acquired directly or reconstructed afterwards. In the first case, colour images are formed under the microscope using a triple, a dual or even a large mono-band filter set, and are acquired using a colour camera (Fig. 1A). In the second case, monochrome images are sequentially recorded using narrow mono-band filter sets, aligned and enhanced when needed, and then superimposed (Fig. 1B).

Fig. 1. - Multiple fluorescent images. A) Indirect immunodetection of cyclin A and 5-methylcytosine. Cyclin A was detected with cyanine 3 using a triple layer biotin-streptavidin system. 5-methylcytosine was detected with monoclonal antibody and anti mouse Igs labelled with rhodol green. Acquisition using a doubleband filter set and a colour camera. The arrow indicates a double labelled cell. B) DNA, BrdU and plasma cells labelling using DNA dye propidium iodide, anti-BrdU, anti-Ig antibodies indirectly revealed by AMCA and FITC. Three successive acquisitions using three mono-band filter sets and a monochrome camera. The cell " $a$ " is BrdU labelled, the cell " $b$ " is Ig positive and corresponds to a plasma cell. C) Multifluorescent bead. Three acquisitions at $\times 40$ magnification using three mono-band filter sets under a recent microscope. D) Multifluorescent bead. Three acquisitions at $\times 40$ magnification using three mono-band filter sets under an old microscope. The shifts are around $2 \mu \mathrm{m}$. E) $X / Z$ image of a multifluorescent bead. Two acquisitions using a $\times 40(\mathrm{NA}=1.3)$ plan-neofluar objective under a CLSM. The shift is around $0.8 \mu \mathrm{m} . \mathrm{F}) X / Z$ image of a multifluorescent bead. Two acquisitions using a $\times 63(\mathrm{NA}=1.4)$ plan-apochromat objective under a CLSM. No shift was observed. G) Confocal images of serial optical sections through a Hela cell labelled by anti-DNA methyl-transferase antibodies revealed by rhodol green. The two sections were separated by $4 \mu \mathrm{m}$ and correspond to the upper and lower part of the cell. In the second section, the dark zone corresponds to the nucleus. The labelling is clearly located in the cytoplasm under the experimental conditions used. H) Three-dimensional reconstruction of a lymphocyte with shaded surface. A piece of cell has been removed in order to see the cyclin A immunolabelling distribution. The arrow indicates the unlabelled nucleolưs. Images were acquired using CLSM at $\times 63(\mathrm{NA}=1.4)$ magnification. I) Three-dimensional reconstruction of a lymphocyte by simulated fluorescence process of a triple labelling. Half of the cell has been removed to see the inside. The cell was triple labelled using Hoechst 33342 DNA dye, anti-cyclin A and anti-p34 ${ }^{\text {cdk1 }}$ antibodies indirectly revealed by cyanine 3 and rhodol green. Images were acquired using CLSM at $\times 63$ $(\mathrm{NA}=1.4)$. J) FISH cytogenetic image. Acquisition at $\times 100$ using triple-band filter set and color camera. The arrow indicates a translocation. 

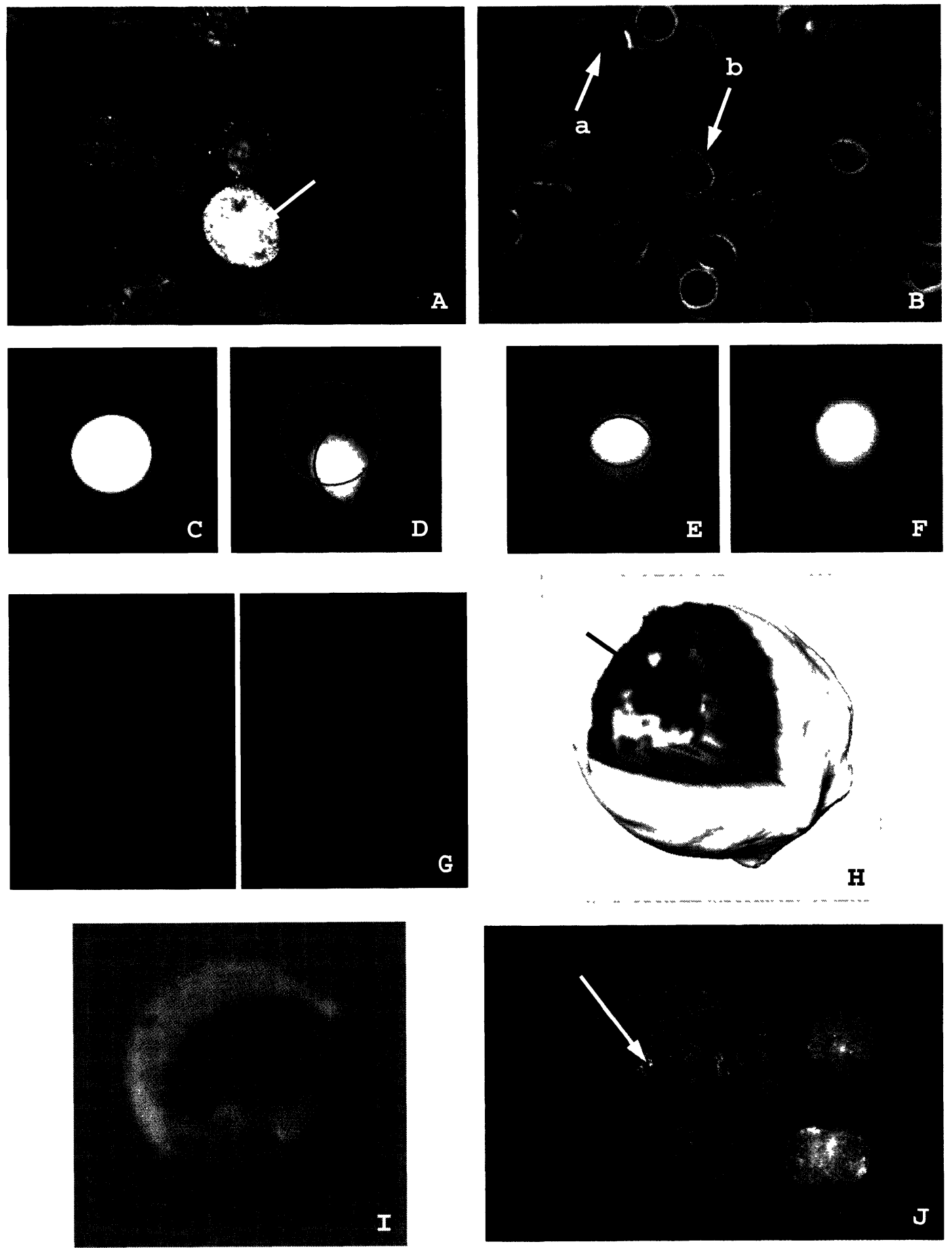
Table I. - Fluorescence filter sets.

\begin{tabular}{|llll|}
\hline Filter set & Excitation & Dichroic mirror & Emission \\
Blue & $360 / 20 \mathrm{~nm}$ & $400 \mathrm{~nm}$ & LP $425 \mathrm{~nm}$ \\
Green & $470 / 20 \mathrm{~nm}$ & $510 \mathrm{~nm}$ & $535 / 25 \mathrm{~nm}$ \\
Red & $538 / 22 \mathrm{~nm}$ & $580 \mathrm{~nm}$ & LP $590 \mathrm{~nm}$ \\
Green/Red & $490 / 20,575 / 30 \mathrm{~nm}$ & $505,600 \mathrm{~nm}$ & $525 / 20,635 / 40 \mathrm{~nm}$ \\
Blue/Green/Red & $400 / 20,495 / 15,570 / 50 \mathrm{~nm}$ & $415,510,590 \mathrm{~nm}$ & $465 / 20,530 / 30,640 / 40 \mathrm{~nm}$ \\
\hline
\end{tabular}

Table II. - Fluorochrome spreading.

\begin{tabular}{|llrrr|}
\hline Fluorochrome & Filter & \multicolumn{3}{c|}{ Camera Channel } \\
& & Blue & Green & \multicolumn{1}{l|}{ Red } \\
Hoechst 33342 & Blue & $87 \pm 0.5^{*}$ & $12 \pm 0.6$ & $1 \pm 0.3$ \\
& B/G/R & $70 \pm 0.5$ & $30 \pm 0.4$ & $0 \pm 0.0$ \\
YOPRO 1 iodide & Green & $1 \pm 0.2$ & $99 \pm 0.2$ & $0 \pm 0.0$ \\
& G/R & $0 \pm 0.1$ & $99 \pm 0.2$ & $1 \pm 0.2$ \\
& B/G/R & $0 \pm 0.3$ & $99 \pm 0.3$ & $1 \pm 0.2$ \\
FITC & Green & $0 \pm 0.1$ & $99 \pm 0.2$ & $1 \pm 0.1$ \\
& G/R & $1 \pm 0.1$ & $74 \pm 0.7$ & $25 \pm 0.9$ \\
& B/G/R & $25 \pm 0.9$ & $59 \pm 1.0$ & $16 \pm 0.4$ \\
Propidium iodide & Red & $0 \pm 0.1$ & $0 \pm 0.0$ & $100 \pm 0.1$ \\
& G/R & $0 \pm 0.1$ & $6 \pm 0.5$ & $94 \pm 0.5$ \\
& B/G/R & $41 \pm 1.3$ & $12 \pm 0.2$ & $47 \pm 1.5$ \\
\hline
\end{tabular}

${ }^{*} \pm$ s.e.m., expressed in $\%$

However, in some cases, colour images can only be recorded after sequential monochrome acquisitions. Thus, the triple immunofluorescence labelling shown in Figure 1B could not be visualized and acquired using the triple band filter. Indeed, due to filter set characteristics (Tab. I), the fluorescence intensity of the label is significantly decreased when multiple rather than monoband filter sets are used, except for green emitting fluorochromes. In the present study, images of single labels were systematically analysed. Fluorescence intensity reached the same level whatever the filter used for green emitting fluorochromes (nuclear Yopro 1 iodide stain, fluorescein isothyocyanate (FITC) immunolabelling). In contrast, nuclei stained with propidium iodide (PI) dye required a four times greater integration time with multiple band filter sets $(=2.4 \mathrm{~s})$ than with the red band filter $(=0.6 \mathrm{~s})$. Likewise, signal strength was drastically reduced for blue emitting fluorochromes. Nuclei stained with Hoechst 33342 dye were acquired during $0.8 \mathrm{~s}$ integration using the triple band filter set and with only a single frame integration using the blue band filter set. The triple filters were mainly designed for fluorescence in situ hybridization (FISH) applications [1] which require reducing the nuclear blue fluorescence in order to keep the green or red fluorescence of DNA probes to be masked.

3.2 Colour Spreading. - Mean intensity percentages observed in the three CCD channels were calculated (Tab. II). Images acquired using mono narrow-band filter sets were mainly observed in one CCD colour channel. Multiple fluorescence images may thus be acquired 
with monochrome cameras and conventional microscopy, or CLSM using such narrow monoband filters with BP and not LP emission filters. Furthermore, triple labelling can simply rise to a multicolour image, assuming that the blue, green or red emitting fluorochromes respectively correspond to the image blue, green or red colour channel (Fig. 1B).

As expected, fluorescence spreading was significantly higher using multiple band filter sets [2] than using a narrow mono-band filter, and colour compensation [3, 4] should be applied. It may be due both to excitation at non-optimum wavelengths [2] and to a wider emission spectrum [4]. However, unexpected high values were observed in the blue channel using the triple-band filter for red and green emitting fluorochromes, and might correspond to a reflection image mixed with the fluorescence image.

3.3 Alignment Discrepancy. - In some cases, monochrome images may have shifted and need to be realigned before being superimposed. Using the recent DMRBE microscope, images of various fluorochromes did not suffer any shifts (Fig. 1C). However, $X / Y$ translations calculated with poly-fluorescent beads were always necessary using an old microscope (Axioplan, Zeiss) (Fig. 1D) and using the CLSM under UV illumination. The image of Figure 1B was acquired in the same conditions that the bead of Figure 1D, but it is shown after shift correction. Moreover, in CLSM, a $800 \mathrm{~nm} Z$ shift occurred between green emitting and red emitting fluorochromes, using the $\times 40$ neofluar (Fig. $1 \mathrm{E}$ ) and an old $\times 40$ plan-apochromat but not with the recent $\times 63$ planapochromat (Fig. 1F). As stressed by other authors [5], chromatic errors are lower with recent than with old microscopes.

3.4 Confocal or Conventional Epifluorescence Microscopy. - In confocal microscopy, fluorescence of out-of-focus structures is stopped by the pinhole aperture, and the $X, Y$, $Z$ resolutions can thus be improved. Serial optical images under various microscope modes can be acquired (Fig. 1G) and 3D image reconstructions become possible [6] (Figs. 1H, I). CLSM has major advantages over conventional microscopy mainly for thick tissue sections or cells when their 3D aspects have been preserved, and at high magnification. This is important for 3D spatial distribution and precise colocalization studies, but not in other cases such as total fluorescence quantification, and routine FISH cytogenetics. For instance, integrated fluorescence intensities of cytocentrifuged cells were measured using an intensified CCD camera [7]. Efficient FISH cytogenetic acquisitions were performed using an integrated colour CCD camera, a triple-band filter set and a software that makes it possible to equilibrate the three CCD channels with respect to each other on line (Fig. 1J). Additionally, softwares applied on series of $Z$ images make it possible to remove the out-of-focus part of the images by numerical deconvolution $[8,9]$.

3.5 Low-Light CCD CAMERAS AND USER InTERfaCE. - The camera features other than sensitivity and colour may be taken into account. Cooled CCD cameras may offer a better resolution but a slower acquisition than intensified cameras (SIT or intensified CCD), thereby making automatic focus and living cell analysis difficult. Thermal noise observed after integration is decreased by the cooling part of CCD and may be corrected by subtracting a background image. Random noise observed when using intensified cameras may be efficiently corrected by averaging a series of successive images. The $1024 \times 1024$ cameras have the advantage of providing a better resolution and the possibility to work at lower magnification, but stored images are four times larger than those obtained using $512 \times 512$ cameras. We feel it worthwhile only in very few applications, when spots or thin details are present and gathered in small zones. An eight-bit depth is mainly used to digitize the fluorescence intensity. However, difficulties were encountered with an excessively range of intensity. 
Judging from our experience over the past few months, in fluorescence microscopy, the key point remains the speed of acquisition and display, and this feature mainly depends on the user interface. The system must be easy to use and must not involve both hardware and software interactions. The possibility to handle cameras (integration time or gain level adjustable for each fluorochrome), epi-fluorescence microscopes (shutter, fluorescence filter set) and CLSMs (laser, emission filter, detector adjustment) through the software interface is already appearing and will probably be developed as needs grow among customers.

\section{Conclusion}

This report illustrates various recent developments in fluorescence microscopy. These new equipments have an interest for specific applications. Multiple band filters make it possible to take photographs and to acquire numerical colour images under the microscope in some applications such as FISH cytogenetics. Compared to photography, numerical image acquisition has the advantage of displaying images on line and of making acquisition easy. CLSM covers the 3D microscopy field. Three-dimensional numerical images may be acquired and further analysed using image analysis softwares. In other various applications, mono-band filters associated with monochrome cameras, still remain the more efficient tool with improvements due to automation.

\section{Acknowledgements}

This study was supported by a grant from ARC. Specimens used during this study were kindly supplied by E. Callet-Bouchu, S. Gazzo, P. Felman (Hematology, Lyon Sud Hospital), M. Ffrench (Edouard Herriot Hospital) and A.M. Manel (Debrousse Hospital). Moreover, the authors would like to thank Cécile Mathieu, Régine Catallo for her technical help and Danny Clausen for reviewing the manuscript.

\section{References}

[1] Joos S., Fink T.M., Ratsch A. and Lichter P., Mapping and chromosome analysis: the potential of fluorescence in situ hybridization, J. Biotechn. 35 (1994) 135-153.

[2] Lowy R.J., Evaluation of triple-band filters for quantitative epifluorescence microscopy, J. Microsc. 178 (1995) 240-250.

[3] Castleman K.R., Colour compensation for digitized FISH images, Bioimaging 1 (1993) 159-165.

[4] Gothot A., Grosdent J.C. and Paulus J.M., A strategy for multiple immunophenotyping by image cytometry: model studies using latex microbeads for seven streptavidin-bound fluorochromes, Cytometry 24 (1996) 214-235.

[5] Florijn RJ., Bonnet J. Vrolijk H., Raap A.K. and Tanke H.J., Effect of chromatic errors in microscopy on the visualization of multi-color fluorescence in situ hybridization, Cytometry 23 (1996) 8-14.

[6] Pawley J.P., Handbook of Biological Confocal Microscopy (Plenum Press Ed, 1995).

[7] Souchier C., Ffrench M., Benchaib M., Catallo R. and Bryon P.A., Methods for cell proliferation analysis by fluorescent image cytometry, Cytometry 20 (1995) 203-209.

[8] Carrington W.A., Lynch R.M., Moore E.D., Isenberg G., Fogarty K.E. and Fay F.S., Superresolution three-dimensional images of fluorescence in cells with minimal light exposure, Science 268 (1995) 14831487.

[9] Shaw P., Deconvolution in 3-D optical microscopy, Histochem. J. 26 (1994) 687-694. 